'Servicio Anestesia Cardiovascular, Hospital Gustavo Fricke. Viña del Mar, Chile.

2Fundación Cardiovascular Dr. Jorge Kaplan Meyer. Viña del Mar, Chile.

${ }^{3}$ Residente de Programa de Especialidad Médica en Cirugía Cardiovascular, Postgrado, Facultad de Medicina. Universidad de Valparaíso. Valparaíso, Chile.

${ }^{4}$ Servicio de Cirugía Cardiovascular, Hospital Gustavo Fricke. Viña del Mar, Chile. anterno de Escuela de Medicina Universidad de Valparaíso, Chile.

Trabajo no recibió financiamiento. Los autores declaran no tener conflictos de interés.

Recibido el 29 de julio de 2019, aceptado el 26 de noviembre de 2019.

Correspondencia a: Manuel Quiroz Flores Álvarez 1532, Viña del Mar, Valparaíso. quirozflores.manuel@gmail.com

\section{Mixoma cardíaco: experiencia de 28 años en resección quirúrgica}

\author{
LORENZO MERELLO ${ }^{1,2}$, VICTORIA ELTON ${ }^{a}$, DIEGO GONZÁLEZ ${ }^{a}$, \\ FELIPE ELGUETA ${ }^{a}$, RODRIGO SALAZAR ${ }^{\mathrm{a}}$, MANUEL QUIROZ ${ }^{3}$, \\ ONEGLIO PEDEMONTE ${ }^{1,2}$, ERNESTO ARANGUIZ ${ }^{2,4}$
}

\section{Cardiac myxomas. Analysis of 78 cases}

Background: The incidence rates of cardiac tumors are low. Aim: To report the clinical presentation of cardiac myxomas and long-term evolution after resection. Material and Methods: Review of a database of surgical patients undergoing surgical resection of a cardiac myxoma in a public hospital between 1990 and 2018. Results: Seventy-eight patients aged $53 \pm 15$ years (65\% females) were included. The most frequent comorbidities were arterial hypertension (40.5\%), hypothyroidism (15\%) and diabetes mellitus (12\%). The main presenting symptoms were dyspnea (33\%), neurological deficit secondary to embolism (30\%) and acute pulmonary edema (5\%). The most common location was the left atrium, in $87 \%$. During surgery, cardiopulmonary bypass and aortic crossclamp times were $50.2 \pm 19.6$ and $33.4 \pm 15.2 \mathrm{~min}$, respectively. One patient died due to severe neurological involvement. Follow-up was completed in seventy-seven patients, with a mean echocardiographic follow-up time of $10.4 \pm 7.7$ years. Thirty-four patients were followed for more than 10 years. Six patients (7.7\%) died during the follow-up and in six patients (7\%) a recurrence was identified. Conclusions: Cardiac myxoma usually has nonspecific symptoms. Surgical excision offers excellent short and long-term results. Complications and recurrence rates are low in non-hereditary myxomas but obligates to perform echocardiographic follow-up for early diagnosis of recurrence.

(Rev Med Chile 2020; 148: 78-82)

Key words: Cardiac Surgical Procedures; Heart Neoplasms; Myxoma; Survival Analysis.

\section{L} os tumores cardíacos primarios son una patología poco común, entre 5\% y $10 \%$ de todos los tumores cardíacos, en este grupo, $80 \%$ son benignos y de estos, más de la mitad corresponde a mixomas. Respecto a su incidencia reportada en cirugía cardiovascular inicialmente se encontraba entre 0,0013 y $0,03 \%$, sin embargo, esto ha aumentado con el diagnóstico ecográfico ${ }^{1-3}$.

Cerca de $90 \%$ de los casos se presentan de forma aislada; 1 a 3\% de los casos son heredados, generalmente en contexto de síndrome de Carney o síndromes mixomatosos ${ }^{4,5}$. Pueden presentarse a cualquier edad, siendo particularmente frecuentes entre la tercera y sexta década de vida, con predominio femenino ${ }^{6}$.

En nuestro medio, el estudio de Becker y cols. describe la experiencia de 30 años en un centro $\mathrm{y}$ encontraron un predominio en mujeres de $64,8 \%$, con localización en aurícula izquierda en $83 \%$ y una sobrevida libre de recurrencia de $97 \%$ a 10 años, lo que se condice con la literatura internacional ${ }^{7}$.

El presente estudio pretende aportar a lo reportado en Chile con nuestra experiencia en el tratamiento de esta entidad, describimos la presentación clínica y los resultados a corto y a largo plazo en términos de sobrevida y complicaciones. 


\section{Materiales y Métodos}

Se realizó un análisis de cohorte retrospectivo descriptivo de los pacientes que fueron sometidos a la resección quirúrgica de un mixoma cardiaco en el Servicio de Cirugía Cardiovascular del Hospital Dr. Gustavo Fricke de Viña del Mar, Chile, entre enero de 1990 y agosto de 2018.

Se obtuvo aprobación por el comité de ética del servicio de salud para la realización de este estudio retrospectivo.

Los datos se obtuvieron de registros electrónicos, protocolos quirúrgicos y de la ficha clínica del hospital. El seguimiento ambulatorio fue clínico y ecocardiográfico. Se obtuvo, además, datos de anatomía patológica y de sobrevida obtenidos del Registro Civil. Se excluyó a pacientes con histología diferente al mixoma.

\section{Procedimiento quirúrgico}

La cirugía se efectuó por esternotomía media con canulación bicava y aórtica en todos los casos. La cardioplegia fue cristalina y sanguínea anterógrada intermitente y el vent mediante incisión de la vena pulmonar superior derecha durante el tiempo de resección del tumor, posteriormente mediante cánula en esta posición y, además, mediante la raíz aórtica.

El abordaje del tumor se realizó principalmente por vía auricular derecha (5 casos), transeptal (55 casos) y en algunos casos directamente por aurícula izquierda (14 casos).

Se realizó cierre primario del defecto en $85 \%$ de los casos y requirieron parche de pericardio autólogo 11 pacientes.

\section{Análisis de datos}

Los resultados se presentan mediante estadística descriptiva, utilizando media como medida de tendencia central y desviación estándar como medida de dispersión para variables continuas. Las variables categóricas son descritas individualmente, mientras que la variable sobrevida se analizó mediante el estimador Kaplan-Meier. Los datos se procesaron mediante el software STATA ${ }^{\circledR}$ versión 11.

\section{Resultados}

Se identificaron 78 pacientes con biopsia compatible con mixoma cardiaco operados entre enero de 1990 y octubre de 2018.

\section{Características de la población}

Del total de pacientes, $51(64,5 \%)$ eran mujeres. La edad promedio fue $53 \pm 15$ años (6-79 años). El antecedente mórbido más frecuente fue la hipertensión arterial, en 32 casos (40,5\%). (Tabla 1).

La presentación clínica predominante fue la disnea, presente en 26 casos (33\%), seguido de embolismo con déficit neurológico en 24 casos $(30 \%)$ y en 14 pacientes fue un hallazgo ecocardiográfico (Tabla 1).

La localización más frecuente fue la aurícula izquierda, con 87,3\% de los casos; en este grupo la ubicación más frecuente fue en el septum interauricular con 55 casos. En aurícula derecha encontramos 5 casos y en ventrículo izquierdo un caso (Tabla 2).

El tamaño promedio del mixoma fue $27 \mathrm{~mm} \pm$ 25, y los pacientes que presentaron embolización cerebral en $100 \%$ de los casos la ubicación del mixoma fue en el septum interauricular.

Tabla 1. Características y presentación clínica

\begin{tabular}{|lcc|}
\hline Características & \multicolumn{2}{c|}{$\mathbf{n}(\%)$ o } \\
promedio (DS)
\end{tabular}


Tabla 2. Datos del procedimiento y seguimiento

\begin{tabular}{|c|c|c|}
\hline \multirow{2}{*}{$\begin{array}{l}\text { Datos del procedimiento } \\
\text { Total }\end{array}$} & \multicolumn{2}{|c|}{$\begin{array}{c}\text { n (\%) o } \\
\text { promedio (DS) }\end{array}$} \\
\hline & \multicolumn{2}{|c|}{$84(100)$} \\
\hline Clamp & \multicolumn{2}{|c|}{$33,4(15,2)$} \\
\hline CEC & \multicolumn{2}{|c|}{$50,2(19,6)$} \\
\hline \multicolumn{3}{|l|}{ Localización } \\
\hline Aurícula izquierda & 69 & $(87,3)$ \\
\hline Septum interauricular & 55 & \\
\hline Pared anterior & 2 & \\
\hline Pared inferior & 7 & \\
\hline Techo y orejuela & 5 & \\
\hline Aurícula derecha & 5 & $(6,3)$ \\
\hline Septum interauricular & 3 & \\
\hline Pared lateral & 2 & \\
\hline Biauricular & 3 & $(3,7)$ \\
\hline Ventrículo izquierdo & 1 & $(1,2)$ \\
\hline
\end{tabular}

Clamp: tiempo de clamp aórtico; CEC: circulación extracorpórea.

\section{Características quirúrgicas}

Se realizaron 84 intervenciones quirúrgicas en un total de 78 pacientes.

El promedio de tiempo de clamp aórtico fue de 33,4 $\pm 15,2$ min y de circulación extracorpórea (CEC) de 50,2 $\pm 19,6 \mathrm{~min}$. La temperatura promedio en CEC fue $36^{\circ} \mathrm{C}$.

Del total de procedimientos, seis fueron reoperaciones $(7 \%)$. En cuatro de estos casos se identificó una recidiva mediante ecografía de control en el seguimiento a 3,4 y 6 años después de la cirugía, respectivamente. Dos pacientes presentaron síntomas durante el seguimiento.

El diagnóstico de mixoma cardiaco fue con- firmado histopatológicamente en todos los casos. (Tabla 2).

\section{Complicaciones}

No presentamos complicaciones quirúrgicas ni reintervenciones de urgencia en este grupo de pacientes.

Se derivaron 7 pacientes $(8,8 \%)$ a rehabilitación debido a accidentes cerebrovasculares previos a la cirugía. En uno de los casos se requirió traslado a otro centro de la red, egresando posteriormente en buenas condiciones.

Solo un paciente falleció durante la hospitalización, debido a compromiso neurológico postoperatorio secundario a accidente cerebrovascular.

\section{Seguimiento a largo plazo}

El seguimiento clínico y ecocardiográfico a largo plazo se documentó en 77 pacientes.

El promedio de años de seguimiento fue de 10,4 \pm 7,7 (0-28 años).

Del total de pacientes, seis fallecieron $(7,7 \%)$ en un promedio de tiempo de $8,8 \pm 6,3$ años después de la cirugía. Las causas de muerte fueron insuficiencia cardiaca congestiva en 2 casos, cáncer vesicular y hepático, un caso, respectivamente, falla multiorgánica en un paciente hospitalizado por accidente vehicular y un suicidio.

La recurrencia detectada por seguimiento ecocardiográfico se logró en 4 pacientes y debido a síntomas que llevaron a estudio en 2 pacientes.

Observamos que a 5 años, la totalidad de los pacientes se encontraban vivos y que a 10 años, esto se mantenía cercano a 95\%. Luego, a los 15 años, la sobrevida llegaba $85 \%$ y se mantenía así hasta los 25 años (Figura 1).

No presentamos diferencias de sobrevida significativas al comparar por sexo a 5, 10 y 15 años.

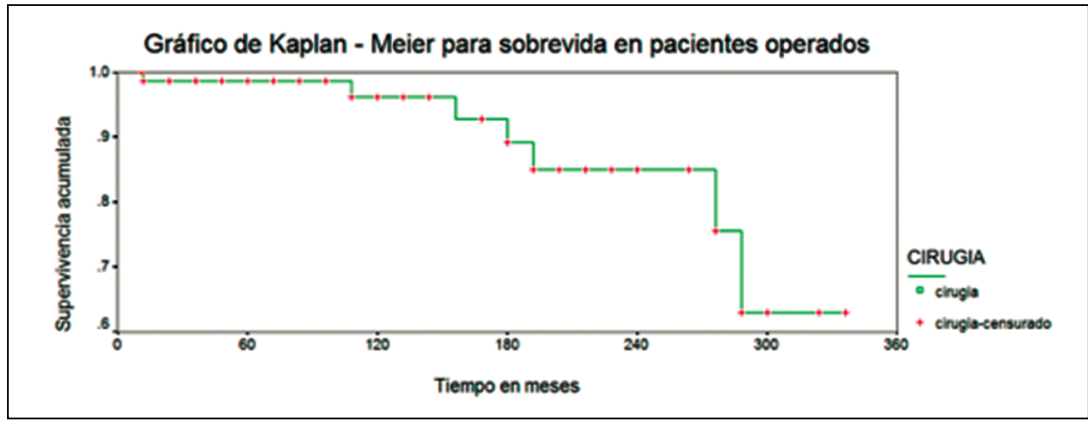

Figura 1. Sobrevida acumulada a largo plazo de pacientes operados de mixomas cardíacos. 


\section{Discusión}

Los mixomas son los tumores cardíacos primarios más frecuentes, sin embargo, su incidencia es baja y en nuestro centro, durante el período de estudio, correspondieron a $0,66 \%$ de las cirugías cardíacas realizadas con CEC. Se consideran tumores benignos, pero con comportamiento potencialmente letal ${ }^{1-7}$.

Nuestra serie mostró que $64,5 \%$ de los pacientes eran mujeres con una edad media de $53 \pm 15$ años, similar a lo encontrado por Becker et al., con $64,8 \%$ de mujeres en su serie, lo que se corresponde a lo reportado a nivel mundial ${ }^{6-10}$.

Los motivos de consulta reportados con mayor frecuencia fueron disnea y edema pulmonar agudo, seguido de síntomas secundarios a embolización sistémica, principalmente al sistema nervioso central y $2,5 \%$ a las arterias de extremidades inferiores. Dos pacientes se presentaron con infarto agudo al miocardio, posiblemente secundario a embolización coronaria. En 18 pacientes, el diagnóstico fue un hallazgo (Tabla 1), resultados similares a los reportados en la literatura (Figura 2) $)^{5-10}$.

Coincidente con la literatura, la localización más frecuente fue la aurícula izquierda $(87,3 \%)$, principalmente el septum interauricular en el $80 \%$ de estos (Tabla 2) ) $^{3,6-10}$.

Encontramos un caso con localización en ventrículo izquierdo, asintomático, detectado por estudio ecocardiográfico, que corresponde a $1 \%$ de nuestra serie y reportado previamente por nuestro equipo ${ }^{11}$.

El diagnóstico por ecocardiografía ha representado un gran aporte para la pesquisa de pacientes

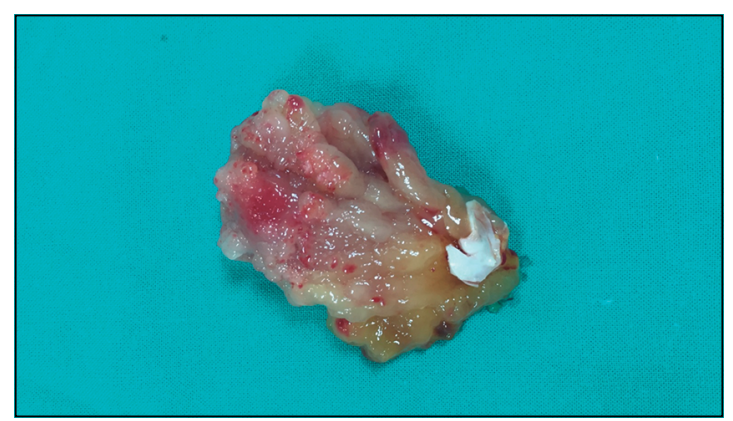

Figura 2. Mixoma auricular con implantación en septum inter-auricular. asintomáticos estudiados por otro motivo o en control por patología cardiovascular como hipertensión arterial, con lo cual el diagnóstico y el volumen de pacientes operados se ha incrementado, ya que este método logra identificar a $90 \%$ de los casos. A su vez, la ecocardiografía 3D permite definir con exactitud la morfología y el tamaño del tumor, así como el grado de obstrucción del flujo sanguíneo, permitiendo una mejor planificación preoperatoria ${ }^{3-7,11}$.

Es relevante mencionar la importancia de la resonancia cardíaca en la caracterización de estos tumores, ya que aporta información respecto a los tejidos adyacentes, composición y vascularización del tumor esperamos que su disponibilidad aumente en beneficio del estudio de nuestros pacientes $^{12}$.

La resección quirúrgica fue realizada por abordaje transeptal en la mayoría de los casos y nuestro equipo coincide con lo publicado por Becker et al., respecto a los beneficios de esta vía.

La cirugía del tumor es el único tratamiento efectivo, eficaz y con buenos resultados a largo plazo, con una tasa de recurrencia que varía entre 1 y $5 \%$ en mixomas esporádicos, en nuestro estudio, la recurrencia fue $5 \%$, sin embargo, atribuimos este porcentaje a 4 pacientes con diagnóstico de mixomatosis hereditaria ${ }^{8-10}$.

El seguimiento se completó en 77 pacientes, donde se observó una sobrevida alta, considerando que el tiempo máximo de seguimiento de 321 meses ( 26 años) donde más de $85 \%$ de los pacientes está vivo. En cuanto a la sobrevida según género, no se encontró una diferencia estadísticamente significativa.

Podemos concluir que los mixomas son una entidad clínica poco frecuente, con síntomas inespecíficos que pueden ser graves. La resección quirúrgica es la única opción de tratamiento, con una baja mortalidad y con excelentes resultados a corto y largo plazo, si bien la recidiva es poco frecuente, el seguimiento ecográfico postoperatorio de largo plazo es importante para pesquisar $y$ tratar de forma oportuna una posible recidiva.

Agradecimientos: Los autores agradecen al personal de pabellón, arsenaleras, perfusionistas, anestesiólogos cardiovasculares, UCI cardiovascular y Servicio de Cirugía Cardiovascular y Cardiología por su dedicación y trabajo constante en beneficio de la salud de nuestros pacientes. 


\section{Referencias}

1. Garatti A, Nano G, Canziani A, Gagliardotto P, Mossuto E, Frigiola A, et al. Surgical excision of cardiac myxomas: twenty years' experience at a single institution. Ann Thorac Surg 2012; 93: 825-31.

2. Burke AP, Gómez-Román JJ, Loire R. World Health Organization: Tumours of the Lung, Pleura, Thymus and Heart 2004; IARC Press.

3. Aggarwal S, Barik R, Sarma T, Iyer V, Sai V, Mishra J, et al. Clinical presentation and investigation findings in cardiac myxomas: new insights from the developing world. Am Heart J 2007; 154: 1102-7.

4. Donato G, Conforti F, Camastra C, Ammendola M, Donato A, Renzulli A. The role of mast cell tryptases in cardiac myxoma: Histogenesis and development of a challenging tumor. Oncol Lett 2014; 8: 379-83.

5. Abu Abeeleh M, Saleh S, Alhaddad E, Alsmady M, Alshehabat M, Bani Ismail Z, et al. Cardiac myxoma: clinical characteristics, surgical intervention, intra-operative challenges and outcome. Perfusion 2017; 32: 686-90.

6. Vroomen M, Houthuizen P, Khamooshian A, Soliman Hamad MA, van Straten AHM. Long-term follow-up of 82 patients after surgical excision of atrial myxomas. Interact CardioVasc Thorac Surg 2015; 21:183-8.

7. Becker P, Ramírez A, Zalaquett R, Moran S, Irarrázaval MJ, Arretz C, et al. Mixoma cardiaco: Caracterización clínica, métodos diagnósticos y resultados alejados del tratamiento quirúrgico durante tres décadas de experiencia. Rev Med Chile 2008; 136: 287-95.

8. Lee K, Kim G, Jung Y, Jeong I, Na K, Oh S. Surgical resection of cardiac myxoma-a 30-year single institutional experience. J Cardiothorac Surg 2017; 12: 18.

9. Shah I, Dearani J, Daly R, Suri R, Park S, Joyce L, et al. Cardiac Myxomas: A 50-Year Experience With Resection and Analysis of Risk Factors for Recurrence. Ann Thorac Surg 2015; 100: 495-500.

10. Jain S, Maleszewski J, Stephenson C, Klarich K. Current diagnosis and management of cardiac myxomas. Expert Rev Cardiovasc Ther 2015; 13: 369-75.

11. Díaz R, Aranguiz E. Mixoma ventricular izquierdo asintomático diagnosticado circunstancialmente por ecocardiografía. Rev Med Chile 2013; 141: 1594-7.

12. Penell DJ, Sechtem UP, Higgins CB, Manning WJ, Pohost GM, Rademakers FE, et al. Clinical indications for cardiovascular magnetic resonance (CMR): Consensus Panel Report. Eur Heart J 2004; 25: 1940-65. 